\title{
Avaliação da sobrecarga do cuidador de pacientes neurológicos cadeirantes adultos
}

\author{
Assessment of the burden of adult wheelchair-bound patients \\ with neurological disabilities on the caregiver
}

Luiz Carlos Boaventura ${ }^{1}$ Heloise Cazangi Borges ${ }^{1}$ Armando Hitoshi Ozaki ${ }^{1}$
${ }^{1}$ Faculdade de Saúde e Ciências da Vida, Centro Universitário Nossa Senhora do Patrocínio. R. Madre Maria Basília 965, Centro. 13300-903 Itu SP Brasil. boaventuraluiz@ hotmail.com
Abstract The scope of this study was to evaluate factors that influence the burden of adult wheelchair-bound patients with neurological alterations on informal caregivers. Sixteen informal caregivers of adult wheelchair-bound patients with neurological alterations were evaluated, using the Zarit Burden Interview (ZBI) scale to evaluate the burden on caregivers, as well as gather data on the care and socio-demographic profile of the caregivers, on the socio-economic data and the degree of functional independence of adult wheelchair-bound patients with neurological alterations. Student's t-test, the one-way ANOVA with Tukey method and Pearson's product moment correlation coefficient were used for data analysis. The results associated lower education level of the caregiver $(p=0.01)$ and lower level of information of the affected pathology ( $p$-value $=0.01$ ) to a heavier burden on the caregiver. The therapeutic and social support provided by the support institutions, such as the Physiotherapy Clinic/School of CEUNSP, was revealed as being important to help caregivers to handle situations in an easier manner. Understanding the factors that influence the burden on the caregiver is important for planning and intervention for this specific population group.

Key words Burden on the caregiver, Informal caregiver, Wheelchair-bound persons, Zarit Burden interview, Quality of life
Resumo O objetivo foi avaliar fatores que influenciam na sobrecarga dos cuidadores informais de pacientes neurológicos cadeirantes adultos. Foram entrevistados 16 cuidadores informais de pacientes neurológicos cadeirantes adultos, utilizando para avaliar sua sobrecarga a escala Zarit Burden Interview (ZBI) e coletados os dados de cuidar e sociodemográficos dos cuidadores, os dados socioeconômicos e o grau de independência funcional dos pacientes neurológicos cadeirantes adultos. Para análise dos dados foram utilizados os testes T-students, ANOVA Unifatorial com método de Tukey e Coeficiente de Correlação de Pearson. Resultados: foram associados, menor grau de escolaridade do cuidador ( $p$-valor $=0,01) e$ menor nível de informação da patologia acometida $(p$-valor $=0,01)$, com maiores sobrecargas. $O$ suporte terapêutico e social dado pelas instituições de apoio, como a Clínica/Escola de Fisioterapia da CEUNSP, demonstrou ser importante para auxiliar os cuidadores a enfrentar situações de forma mais tranquila. O conhecimento de fatores que podem influenciar a sobrecarga dos cuidadores é importante para o planejamento e a intervenção desse público específico.

Palavras-chave Sobrecarga do cuidador, Cuidador informal, Cadeirante, Zarit Burden Interview, Qualidade de vida 


\section{Introdução}

Cuidador pode ser uma pessoa da família ou sociedade que presta serviços a um indivíduo de qualquer idade que esteja necessitando de cuidados, devido a limitações físicas ou mentais, podendo ser remunerado ou não por este auxílio. A sua função é acompanhar e auxiliar a pessoa a se cuidar, fazendo somente as atividades que ela não consiga fazer sozinha. É importante ficar claro que não fazem parte da rotina do cuidador, técnicas e procedimentos identificados de profissões legalmente estabelecidas, como as da área de enfermagem ${ }^{1}$.

Para efeito de classificação dos cuidadores, utiliza-se a classificação do Ministério de Estado da Previdência e Assistência Social (MPAS) que os dividem em: profissional, formal e informal. Sendo:

- Profissional: indivíduo que possui formação com diploma conferido por instituição de ensino reconhecida oficialmente e que presta assistência profissional ao idoso, à família e à comunidade. O perfil do cuidador profissional é ter graduação e treinamento específico em cuidados em instituições oficialmente reconhecidas.

- Formal: indivíduo capacitado para auxiliar a pessoa que apresenta limitações para realizar as atividades e as tarefas da vida cotidiana, fazendo a união entre a pessoa cuidada, a família e os serviços de saúde ou da comunidade, sendo geralmente remunerado. O perfil do cuidador formal é ter ensino fundamental, ser maior de idade e submetido a treinamento específico, ministrado por instituição reconhecida.

- Informal: indivíduo que presta cuidados à pessoa cuidada no domicílio, com ou sem vínculo familiar, não sendo remunerado. $\mathrm{O}$ perfil do cuidador informal é ser pessoa de ambos os sexos, pertencentes à família ou não, que tem uma pessoa que necessita de ajuda em casa e identifica-se com as atividades pertinentes ${ }^{2}$.

A família é quem geralmente assume a responsabilidade pela saúde de seus membros, formando a unidade que presta cuidado, buscando promover a saúde, a prevenção e o tratamento de patologias, sendo também a responsável pela formação tanto individual quanto social de seus membros. Exercendo, portanto, funções nas áreas educacional, econômica, doméstica e de cuidados com a saúde de seus membros, além de recreação, socialização e afeição, porém o familiar que assume a responsabilidade de cuidar deve ser integrante da família e não elemento a parte, único e exclusivamente responsável pelo cuidar. Vale lembrar que a família que tem um membro doente deve ser vista de forma ampla e aberta, um sistema que interage com outros de prestação de cuidados à saúde ${ }^{3}$.

A escolha do cuidador informal, de pessoas idosas, está relacionada a vários fatores, como grau de parentesco (maioria cônjuges), gênero (geralmente mulher), proximidade física (quem mais convive) e proximidade afetiva (cônjuge, pais e filhos). Na maioria das vezes, o cuidado é feito por uma única pessoa da família, que se torna cuidador principal ou referência, por maior disponibilidade, instinto, vontade ou capacidade, geralmente sem formação básica orientada ${ }^{4}$.

$\mathrm{O}$ ato de cuidar é complexo, pois o cuidador e a pessoa a ser cuidada apresentam sentimentos contraditórios e diversificados, como exemplo: culpa, raiva, medo, confusão, estresse, tristeza, bem como alegria, gratidão, sonhos e fé. Muitas vezes esses sentimentos podem aparecer de forma isolada ou mesmo juntas, o que é bastante normal ao longo do dia e fazem parte da relação do cuidador e da pessoa cuidada. É fundamental que se perceba entre as duas partes as reações e os sentimentos que afloram, para melhor compreensão de um lado e do outro, buscando formas para resolver e planejar uma melhor convivência, mantendo a integridade física e emocional, além da qualidade de vida tanto do cuidador como da pessoa a ser cuidada ${ }^{5}$.

O impacto de cuidar de uma pessoa dependente, sujeito a uma condição crônica, é descrito pelo termo burden, burdensome ou, raramente, burnout. Esses termos foram primeiramente publicados por Zarit, em 1980, e incluíam os problemas físicos, psicológicos ou emocionais, sociais, financeiros vividos por famílias cuidadoras, representados tanto por aspectos subjetivos quanto objetivos, advindos do impacto do cuidar. Usaremos o termo burden que vem da língua inglesa, usada para descrever aspectos negativos associados aos cuidadores, geralmente, informal e familiar, de indivíduos doentes que é semelhante aos termos impacto, sobrecarga, fardo e interferência. E essa sobrecarga pode ser definida como qualidade ou quantidade de demanda que vai além da capacidade de desempenho do cuidador, por insuficiência técnica ou de tempo e que a pressão e a responsabilidade no trabalho proporcionam, principalmente, o aparecimento da exaustão emocional ${ }^{6}$.

A sobrecarga do cuidador é considerada então como uma perturbação resultante do trabalho em lidar com a dependência física e incapacidade mental do sujeito que necessita de atenção e cuidados, como de um conjunto de problemas físicos, mentais e socioeconômicos que sofrem os 
cuidadores de pessoas com enfermidades, afetando atividades cotidianas, relacionamentos sociais e equilíbrio emocional ${ }^{7}$.

Baseando-se nisto, o objetivo deste estudo foi avaliar fatores que influenciam na sobrecarga dos cuidadores informais de pacientes neurológicos cadeirantes adultos, descrevendo as características de ambos, bem como o grau de independência funcional dos pacientes - e o de sobrecarga dos primeiros - atendidos na Clínica/Escola de Fisioterapia do Centro Universitário Nossa Senhora do Patrocínio (CEUNSP).

Foi importante não só mensurar o grau de sobrecarga do cuidador informal, mas também entender o sujeito que dependia de cuidados, relacionando-os. No estudo, sendo o paciente neurológico cadeirante adulto, ou seja, o indivíduo que teve qualquer lesão no funcionamento neural e que pode apresentar três efeitos distintos: 1. perda, déficit, abolição ou destruição (total ou parcial, transitória ou permanente) de determinada capacidade funcional; 2: exacerbação, liberação ou desinibição de função normalmente controlada, refreada ou inibida; e, 3: irritação ou excitação, causando efeito inexistente. Sendo exemplos de déficit ou perda funcional: a paralisia, a hipoestesia, a anestesia, a arreflexia, o declínio da inteligência ou da memória; e de liberação ou exacerbação: hipertonia, hiperreflexia, movimentos anormais, disestesia; por fim exemplos de efeito irritativo: dor por irritação de nervo periférico, crise epiléptica. Onde os sinais e sintomas resultam, normalmente, mais da localização da lesão do que da natureza da mesma, como exemplo: uma pessoa que sofreu acidente vascular cerebral e um outro que tem um tumor no córtex motor primário produzem ambos, basicamente, o mesmo déficit neurológico, ou seja a mesma hemiplegia, pois, apesar de terem etiologias diferentes, possuem basicamente os mesmos sinais e sintomas devido ao local de lesão no sistema nervoso ${ }^{8}$.

Isto nos indica que não basta conhecer somente a patologia neurológica do paciente, mas que foi necessário saber o tipo e o local de lesão que a patologia acometeu, tentando posteriormente mensurar o grau de déficit funcional do indivíduo, o qual estava relacionado com três importantes conceitos: autonomia, independência e dependência do sujeito. Sendo:

- Autonomia: definida como autogovernar, liberdade de agir e tomar decisões.

- Independência: definida como ser capaz de realizar as atividades sem ajuda de outra pessoa.

- Dependência: definida como não ser capaz de realizar as atividades cotidianas sem ajuda de outra pessoa ${ }^{5}$.
Alguns indivíduos neurológicos cadeirantes podem ser dependentes (incapazes de realizar determinadas tarefas sozinhas), embora tenham total autonomia (capacidade de decisão). Bem como, podem ter ambas comprometidas, tanto a capacidade de decidir como a de executar, devido a comprometimentos físicos e mentais. Vale salientar que mesmo a pessoa sendo dependente, nem sempre ela perde sua autonomia e isso deve ser respeitado, não devemos privar a pessoa de tomar decisões, devido a encontrar-se num estado de limitação física, não sendo capaz de realizar total ou parcialmente uma determinada tarefa. Para isso, foi necessário verificar o grau de independência funcional desses indivíduos, ou seja, qual a necessidade de ajuda ou assistência esta pessoa demanda, seja no auxilio, supervisão ou preparo para realização de tarefas motoras ou cognitivas e se esta demanda estava associada também com o grau de sobrecarga do cuidador informal.

\section{Método}

Foi realizado um estudo clínico, transversal e analítico na Clínica/Escola de Fisioterapia do CEUNSP em Itu/SP, por um estudante graduando em fisioterapia, sob a supervisão e orientação de dois docentes, um especializado em neurologia e outro em clinica médica, sendo todos vinculados à instituição proponente.

O pesquisador teve acesso aos prontuários dos pacientes atendidos no setor de fisioterapia neurológica, no período de janeiro de 2014 até maio de 2015, com o intuito de identificar os sujeitos do estudo e posteriormente solicitar sua colaboração na pesquisa, adotando os seguintes critérios de inclusão: pacientes que tinham diagnóstico clínico de patologia neurológica, com idade igual ou maior que 18 anos completos, fazendo uso de cadeira de rodas. O convite para participação da pesquisa foi realizado, através de contato telefônico ou pessoalmente.

No período foram atendidos na Clínica/Escola do CEUNSP, no setor de Fisioterapia Neurológica, 164 pacientes, sendo destes 117 neurológicos adultos e 47 neurológicos adolescentes, crianças e lactentes. Dos 117 pacientes neurológicos adultos, 32 eram cadeirantes.

Todos os 32 pacientes neurológicos cadeirantes adultos foram selecionados para participar da pesquisa, bem como seus respectivos cuidadores. Entretanto, não foi possível comunicar e convidar seis deles; também foram excluídos seis, um deles devido a falecimento, três por não terem cuidadores e dois por terem cuidadores formais, 
ou seja, estes recebiam qualquer fonte de remuneração para cuidar.

Devido à necessidade de assinar o Termo de Consentimento Livre e Esclarecido e comparecer à Clinica/Escola de Fisioterapia do CEUNSP com agendamento prévio para entrevista, nos meses de maio e junho de 2015, a amostra de 20 pacientes neurológicos cadeirantes adultos e 20 cuidadores reduziu-se para $16 \mathrm{em}$ ambos casos.

Foi realizada entrevista somente dos cuidadores informais principais, ou seja, o cuidador que passava mais tempo cuidando e tinha a maior responsabilidade sobre o paciente neurológico cadeirante adulto, tendo a entrevista duração aproximada de 20 minutos.

A pesquisa foi submetida e aprovada pelo Comitê de Ética em Pesquisa da Faculdade de Medicina da Universidade de São Paulo - FMUSP.

Os instrumentos e as escalas de avaliação utilizados neste estudo não apresentavam nenhum risco ao entrevistado e foram para a:

- Avaliação do perfil dos cuidadores informais, o Instrumento de Avaliação para Caracterização do Cuidador Informal, elaborado pelo pesquisador junto com os orientadores especializados em neurologia e clinica média, que permitiu conhecer os dados dos cuidadores informais nos aspectos: sociodemográficos (idade em anos completos, gênero, grau de parentesco, grau de escolaridade em anos de estudo, estado civil, se residia ou não com o paciente neurológico cadeirante, se trabalhava e/ou estudava) e do cuidar (conhecimento sobre a patologia do paciente, conhecimento sobre o cuidar, se possuía curso e/ ou treinamento para cuidar, tempo que dedicava semanalmente e diariamente ao cuidado do paciente, tempo total como cuidador do paciente em meses, se recebia ajuda de alguém e de quem).

- Avaliação do perfil dos pacientes neurológicos cadeirantes adultos, o Instrumento de Avaliação para Caracterização do Paciente Neurológico Cadeirante Adulto, elaborado pelo pesquisador junto com os orientadores especializados em neurologia e clinica médica, que permitia conhecer dados dos cuidadores informais nos seguintes aspectos: sociodemográficos (idade em anos completos, gênero, grau de escolaridade em anos de estudo, estado civil, diagnóstico neurológico, tempo como cadeirante e quantidade de pessoas que residiam junto na mesma moradia) e dado econômico (renda mensal familiar e per capita: renda mensal familiar/número de indivíduos que residiam no domicílio).

- Avaliação de sobrecarga dos cuidadores informais dos pacientes neurológicos cadeirantes, utilização da escala Zarit Burden Interview (ZBI) que foi traduzida e validada para a cultura brasileira por Scazufca9. Composto por um questionário com vinte e duas questões, com objetivo de avaliar a sobrecarga que era percebida pelo cuidador e que refletia como a pessoa se sentia ao cuidar de outra, englobava as áreas de saúde, vida social e pessoal, situação financeira, bem-estar emocional e relacionamento interpessoal. Sendo utilizada para avaliar a sobrecarga dos cuidadores de indivíduos com incapacidades metal e física ${ }^{10}$. As questões referentes aos itens 1 ao 21 eram pontuadas numa escala de 0 a 4 pontos, representados da seguinte forma: nunca (0), raramente (1), algumas vezes (2), frequentemente (3) e sempre (4); na questão 22, eram pontuadas de 0 a 4 pontos como: nem um pouco (0), um pouco (1), moderadamente (2), muito (3) e extremamente (4). Todas as perguntas deveriam ser obrigatoriamente pontuadas e cada pontuação indicava a frequência que cada afirmação afetava o cuidador, o total da escala foi obtido pelo somatório de todos os 22 itens, variando de 0 a 88 . Em que as maiores pontuações obtidas correspondiam a maior sobrecarga do cuidador, sendo classificado segundo estudo internacional ${ }^{11}$ e nacional ${ }^{12} \mathrm{em}$ : ausência de sobrecarga de 0 a 20, leve a moderada sobrecarga de 21 a 40, moderado a severa sobrecarga de 41 a 60 e intensa sobrecarga de 61 a 88.

- Avaliação do grau de independência funcional para adultos, a utilização da escala Medida de Independência Funcional (MIF), traduzida e validada no Brasil por Ribeiro et al. ${ }^{13}$, em 2004, cujo objetivo foi verificar o desempenho da pessoa para a realização de um conjunto de 18 tarefas, referentes às subescalas de autocuidado, controle esfincteriano, transferências, locomoção, comunicação e cognição social. Descrevendo dois domínios, sendo motor e o cognitivo, diferenciando a independência funcional dos pacientes de forma objetiva e quantitativa, mensurando o grau de solicitação de cuidados ao paciente, mostrando as principais necessidades de ajuda ou assistência. Cada uma das atividades foi avaliada e recebia uma pontuação de 1 a 7, sendo classificadas em: assistência total (1); assistência máxima (2); assistência moderada (3); assistência mínima (4); supervisão, estímulo ou preparo (5); independência modificada (6) e independência completa (7). Em que a pontuação total variava de 18 a 126, com as menores correspondendo a maior dependência, sendo classificado em: dependência total tendo 18 pontos, dependência máxima a moderada de 19 a 60 pontos, dependência mínima a supervisão, estímulo ou preparo de 61 a 103 e independência modificada a independência completa de 104 a 126 pontos. 
Após a coleta dos dados foi realizada a apuração e a consolidação em uma planilha do programa Microsoft Excel 2010 e feitas as análises estatísticas dos dados.

Inicialmente foram realizadas as análises descritivas referentes às características dos cuidadores informais, as características dos pacientes cadeirantes neurológicos adultos, o grau de sobrecarga dos cuidadores informais e o grau de independência funcional dos pacientes neurológicos cadeirantes adultos ${ }^{14}$. Verificaram-se, também, possíveis associações da sobrecarga dos cuidadores informais em relação às variáveis, dos cuidadores informais e dos pacientes neurológicos cadeirantes adultos, através da pontuação da Escala ZBI.

As associações entre as médias da pontuação total da Escala ZBI (variável quantitativa) e as variáveis qualitativas com duas categorias, dos cuidadores informais e dos pacientes neurológicos cadeirantes adultos, foram calculadas através do teste T-Student ${ }^{15}$. As associações entre a média da pontuação total da Escala ZBI e as variáveis qualitativas com três ou mais categorias, dos cuidadores informais e dos pacientes neurológicos cadeirantes adultos, foram verificadas através da análise de variância (ANOVA - Unifatorial). No caso do teste de análise de variância (ANOVA - Unifatorial) ser significativo ( $<<0,05)$, ou seja, haver a diferença em pelo menos um grupo; neste caso, foi realizada a análise de comparação múltipla, através do método de Tukey, para saber em quais grupos existiam essas diferenças ${ }^{15}$. As associações entre a pontuação total da Escala ZBI (variável quantitativa) e o score das variáveis quantitativas numéricas, dos cuidadores informais e dos pacientes neurológicos cadeirantes adultos, foram calculadas pelo Coeficiente de Correlação de Pearson (r) que varia de -1 a 1; no qual o sinal indicava a direção positiva ou negativa do relacionamento e o valor do coeficiente sugeria a força da relação entre as variáveis; quanto mais próximo de zero era o valor do coeficiente, menor era a força da relação entre as variáveis; e opostamente, quanto mais próximo de 1 era o valor do coeficiente (independente do sinal), maior era o grau de correlação entre as variáveis; sendo 1 (independente do sinal) correlação perfeita e 0 sem correlação ${ }^{16}$. Utilizou-se a classificação da magnitude dos valores do Coeficiente de Correlação de Pearson (r), sendo (independente do sinal): $r=0,10$ até 0,30 (fraca correlação), $r=$ 0,40 até 0,60 (moderada correlação) e $r=0,70$ até 1 (forte correlação), conforme Dancey e Reidy ${ }^{16}$.

O nível de significância para todos os testes estatísticos foi fixado em $5 \%$.
Foram utilizados os testes estatísticos paramétricos, pela aderência na análise da distribuição normal da pontuação da Escala ZBI, calculadas por meio do teste de Shapiro-Wilks, no qual: $\mathrm{W}_{\text {calculado }}=0,899>\mathrm{W}_{0,05: 16}=0,887$ e p-valor $=0,07>\alpha=0,05^{17}$.

Utilizou-se o programa Action, versão 2.9 para realização dos cálculos estatísticos ${ }^{17}$.

\section{Resultado}

A amostra dos 16 cuidadores informais de pacientes neurológicos cadeirantes adultos caracterizou-se por onze do gênero feminino e cinco do masculino, com idade média de $53 \pm 11$ anos, mínima de 31 e máxima de 73.

Todos os cuidadores informais eram membros da família, sendo sete cônjuges, quatro pais, dois filhos(as), dois netos(as) e um(a) irmão(ã). Eram 15 casados e 14 residiam com a pessoa a ser cuidada. Dos cuidadores informais 11 não trabalhavam, pois oito eram donas de casa, dois aposentado(s) ou pensionista(s) e um por outros motivos; 14 não estudavam, somente dois estavam cursando alguma instituição de ensino. Em relação à escolaridade, onze tinham até oito anos de estudo, três de 9 a 12 e dois acima de 13 .

Sobre o conhecimento da patologia acometi$\mathrm{da}$, sete se consideravam bem informados, quatro muito bem informados, três pouco e insuficiente, e dois pouco e suficiente. E em relação sobre o conhecimento de como cuidar, metade consideravam-se bem informada, cinco pouco e suficiente, e três muito bem. Sendo que 13 dos cuidadores não tinha curso e nem treinamento para cuidar, 15 contavam com ajuda de outras pessoas para cuidar, em que seis contavam sempre, cinco às vezes e quatro quase sempre. Quando a ajuda era solicitada, era feita apenas por parentes próximos, não sendo considerado na entrevista o atendimento e a ajuda da Clinica/Escola do CEUNSP.

Em relação ao tempo dedicado a cuidar, todos os cuidadores dedicavam os cinco dias da semana, tendo uma média de $20 \pm 7$ horas por dia, o tempo mínimo foi de 6 horas/dia e o máximo de 24 horas/dia, não houve alteração no tempo dedicado em horas/dia nos dias da semana e nos finais de semana, sendo praticamente o mesmo dedicado na semana e nos finais de semana, 14 cuidadores dedicavam também os dois dias do final de semana para cuidar e somente dois dedicavam um dia ou nenhum dia no final de semana. O tempo mínimo na função de cuidador informal foi de dois meses e o máximo 408 meses 
(32 anos), neste caso os dados não apresentaram uma tendência central, sendo muito divergentes uns para os outros.

O grau de sobrecarga dos cuidadores informais, conforme Tabela 1, demonstrou que nove cuidadores dos cuidadores informais tinha ausência de sobrecarga, cinco a tinham de leve a moderada e dois a tinham de moderado a severa, não houve nenhum cuidador com intenso grau de sobrecarga.

Ao analisarmos a sobrecarga da amostra dos cuidadores informais em relação às suas variáveis qualitativas, conforme Tabela 2, verificamos que a sobrecarga não estava associada ao: gênero, feminino ou masculino ( $\mathrm{p}$-valor $=0,75$ ); se residiam ou não, com a pessoa que cuidavam ( $\mathrm{p}$-valor = $0,25)$; se trabalhavam ou não ( $\mathrm{p}$-valor $=0,26$ ); se estudavam ou não ( $\mathrm{p}$-valor $=0,85)$; se tinham ou não, curso ( $p$-valor $=0,57)$ ou treinamento para cuidar ( $p$-valor $=0,76)$; ou informações sobre como cuidar ( $\mathrm{p}$-valor $=0,13$ ); pois nesses casos não houve uma diferença significativa ( $\mathrm{p}$-valor $<0,05)$ da sobrecarga entre a ou as categoria(s). Porém houve associação da sobrecarga desses cuidadores informais em relação ao seu grau de escolaridade ( $\mathrm{p}$-valor $=0,01)$, tendo uma diferença significativa, entre 1 e 4 anos de estudo e 13 ou mais anos de estudo ( $p$-valor $=0,02)$, e entre 1 a 4 anos de estudo e 5 a 8 anos de estudo (p-valor $=0,01$ ); havendo maior sobrecarga de 1 a 4 anos de estudo (média $=38$ pontos) em relação a 5 a 8 anos (média $=19$ pontos), e maior sobrecarga de 1 a 4 anos de estudo (média $=38$ pontos) em relação a 13 ou mais anos de estudos (média $=$ 13 pontos). Também houve associação da sobrecarga em relação ao nível de conhecimento do cuidador informal sobre a patologia acometida ao paciente neurológico cadeirante adulto ( $\mathrm{p}$ valor $=0,01)$, tendo uma diferença significativa

Tabela 1. Distribuição da frequência do grau de sobrecarga dos cuidadores informais, segundo Escala ZBI ${ }^{9}$. Itu-SP, Brasil - 2015.

\begin{tabular}{lrr}
\hline \multicolumn{1}{c}{ Variável Avaliada } & fi & \multicolumn{1}{c}{$\%$} \\
\hline Grau de sobrecarga dos cuidadores & & \\
informais $^{*}$ & & \\
$\quad$ Ausente (0 a 20 pontos) & 9 & 56,25 \\
Leve a Moderado (21 a 40 pontos) & 5 & 31,25 \\
Moderado a Severo (41 a 60 pontos) & 2 & 12,50 \\
Intenso (61 a 88 pontos) & 0 & 0,00 \\
Total & 16 & 100,00 \\
\end{tabular}

*Avaliada pela pontuação total da Escala ZBI, conforme classificação internacional ${ }^{11}$ e nacional ${ }^{12}$. entre pouco e insuficiente informação para bem informado ( $p$-valor $=0,03$ ) e entre pouca e suficiente informação para bem informado ( $\mathrm{p}$-valor $=0,02$ ), havendo maior sobrecarga de pouco e insuficiente informação (média $=39$ pontos) em relação a bem informado (média $=17$ pontos), e maior sobrecarga entre pouco e suficiente informação (média $=34$ pontos) em relação a bem informado (média $=17$ pontos). Não foi possível fazer a analise de associação da sobrecarga em relação ao estado civil, grau de parentesco e nem se recebiam ou não ajuda, pois os dados da amostra não tiveram aderência aos requisitos dos testes, sendo inconsistentes para fazer as considerações e as significações.

Ao analisarmos a sobrecarga da amostra dos cuidadores informais em relação às suas variáveis quantitativas de idade e tempo como cuidador, conforme Tabela 3, verificamos que a sobrecarga tinha uma relação positiva com a idade dos cuidadores informais, mas que essa correlação era fraca $(r=0,26)$, ou seja, apesar da idade elevada estar relacionada com o aumento da pontuação total da escala ZBI de sobrecarga e consequentemente da sobrecarga, a correlação entre sobrecarga e idade era fraca; opostamente, a relação entre a sobrecarga e o tempo como cuidador teve um direcionamento negativo $(\mathrm{r}=-0,16)$, ou seja, o aumento do tempo como cuidador estava relacionado com a diminuição da pontuação total da escala ZBI de sobrecarga, mas a correlação também foi fraca, $r$ entre 0,10 a 0,30 (independente do sinal).

Referente aos pacientes neurológicos cadeirantes adultos tratados na Clinica/Escola do CEUNSP, a amostra apresentou-se igual em gênero masculino e feminino com idade média de $57 \pm 20$ anos, a idade mínima foi de 26 anos e a máxima de 92 anos. Apresentando-se nove sem uma vida conjugal, sendo: quatro solteiros, três viúvos, um(a) separado(a) e um(a) divorciado(a) ou desquitado(a); e sete casados. O tempo mínimo como cadeirante foi de seis meses e o máximo de 120 meses (10 anos), neste caso, os dados não apresentaram uma tendência central, sendo muito diferente o tempo como cadeirante de uns para os outros. Em média, residiam com $3 \pm 2$ pessoas no mesmo domicílio, mínimo de uma e máximo de sete. Em relação à escolaridade, metade tinha de 5 a 8 anos de estudo, três de 1 a 4 anos, dois eram analfabetos, dois tinham de 9 a 12 anos e um tinha acima de 13 anos de estudo.

$\mathrm{Na}$ questão econômica, tinham uma renda mensal familiar média de $\mathrm{R} \$ 2.616,88 \pm 1.344,35$; mínima de $\mathrm{R} \$ 790,00$ e máxima de $\mathrm{R} \$$ 5.530,00; a renda per capita (renda mensal/número de in- 
Tabela 2. Resultado da análise entre a sobrecarga dos cuidadores informais e as variáveis qualitativas dos cuidadores informais. Itu-SP, Brasil - 2015.

\begin{tabular}{|c|c|c|c|c|}
\hline \multirow[b]{2}{*}{ Variáveis Independentes } & \multicolumn{4}{|c|}{ Variável Dependente (Sobrecarga do Cuidador Informal) } \\
\hline & n $(\%)$ & Média* $^{*}$ & $\begin{array}{l}\text { Desvio } \\
\text { Padrão* }\end{array}$ & p-valor \\
\hline \multicolumn{5}{|l|}{ Gênero } \\
\hline Feminino & $11(68,75)$ & 22,91 & 12,72 & $0,75^{* *}$ \\
\hline Masculino & $5(31,25)$ & 25,00 & 9,30 & \\
\hline \multicolumn{5}{|l|}{ Grau de Escolaridade } \\
\hline De 1 a 4 anos & $4(25,00)$ & 38,00 & 8,87 & $0,01^{* * *}$ \\
\hline De 5 a 8 anos & $7(43,75)$ & 19,14 & 8,59 & \\
\hline De 9 a 12 anos & $3(18,75)$ & 21,67 & 6,66 & \\
\hline De 13 anos ou mais & $2(12,50)$ & 13,00 & 2,83 & \\
\hline \multicolumn{5}{|l|}{ Reside com a pessoa a ser cuidada } \\
\hline Sim & $14(87,50)$ & 22,29 & 9,82 & $0,25^{* *}$ \\
\hline Não & $2(12,50)$ & 32,50 & 23,34 & \\
\hline \multicolumn{5}{|l|}{ Trabalha } \\
\hline Sim & $5(31,25)$ & 27,83 & 10,02 & $0,26^{* *}$ \\
\hline Não & $11(68,75)$ & 21,00 & 13,42 & \\
\hline \multicolumn{5}{|l|}{ Estuda } \\
\hline Sim & $2(12,50)$ & 22,00 & 12,01 & $0,85^{* *}$ \\
\hline Não & $14(87,50)$ & 23,79 & 9,90 & \\
\hline \multicolumn{5}{|l|}{ Teve Curso } \\
\hline Sim & $3(18,75)$ & 20,00 & 12,29 & $0,57^{* *}$ \\
\hline Não & $13(81,25)$ & 24,38 & 7,81 & \\
\hline \multicolumn{5}{|l|}{ Teve Treinamento } \\
\hline Sim & $3(18,75)$ & 21,67 & 10,60 & $0,76^{* *}$ \\
\hline Não & $13(81,25)$ & 24,00 & 12,05 & \\
\hline \multicolumn{5}{|l|}{ Informado da patologia acometida } \\
\hline Pouco e insuficiente & $3(18,75)$ & 34,33 & 6,11 & $0,01^{* * *}$ \\
\hline Pouco e suficiente & $2(12,50)$ & 39,00 & 14,14 & \\
\hline Bem informado & $7(43,75)$ & 16,57 & 7,55 & \\
\hline Muito bem informado & $4(25,00)$ & 20,00 & 6,98 & \\
\hline \multicolumn{5}{|l|}{ Informado sobre como cuidar } \\
\hline Pouco e suficiente & $5(31,25)$ & 30,80 & 14,11 & $0,13^{* * *}$ \\
\hline Bem informado & $8(50,00)$ & 22,50 & 9,32 & \\
\hline Muito bem informado & $3(18,75)$ & 14,33 & 4,93 & \\
\hline
\end{tabular}

"Avaliada pela pontuação total da Escala ZBI'; ${ }^{* *}$ Teste T-Students, ${ }^{* * *}$ Teste ANOVA Unifatorial.

Tabela 3. Resultado da análise entre a sobrecarga dos cuidadores informais e as variáveis quantitativas de idade e tempo como cuidador, dos cuidadores informais. Itu-SP, Brasil - 2015.

\begin{tabular}{lcc}
\hline & \multicolumn{2}{c}{$\begin{array}{c}\text { Sobrecarga do } \\
\text { Cuidador Informal }\end{array}$} \\
\cline { 2 - 3 } \multicolumn{1}{c}{$\begin{array}{c}\text { Variáveis } \\
\text { Avaliadas }\end{array}$} & $\begin{array}{c}\text { Coeficiente } \\
\text { de Correlação }\end{array}$ & $\begin{array}{c}\text { palor } \\
\text { de Pearson }\end{array}$ \\
\hline Idade (em anos completos) & $\mathrm{r}=0,26$ & 0,33 \\
Tempo como cuidador (meses) & $\mathrm{r}=-0,16$ & 0,55 \\
\hline
\end{tabular}

*Avaliada pela pontuação total da Escala ZBI 9 . divíduos residentes no domicílio) foi em média de $\mathrm{R} \$ 693,31 \pm 317,43$; mínima de $\mathrm{R} \$ 395,00$ e máxima de $\mathrm{R} \$ 1.382,50$.

Em relação ao grau de independência funcional, pela pontuação total da Escala de Avaliação MIF, conforme Tabela 4, apresentaram-se doze com dependência máxima a dependência moderada e quatro com dependência mínima a supervisão, estímulo ou preparo.

Ao analisarmos a sobrecarga da amostra dos cuidadores informais em relação às variáveis quantitativas dos pacientes neurológicos cadeirantes adultos, conforme Tabela 5, verificamos que a sobrecarga tinha uma relação positiva com 
a idade $(\mathrm{p}=0,10)$ e o tempo como cadeirante ( $\mathrm{p}$ $=0,09)$, ou seja, quando os pacientes tinham elevada idade e elevado tempo como cadeirantes a sobrecarga dos cuidadores informais era também elevada; já a relação entre renda mensal familiar $(r$ $=-0,03)$, renda per capita $(r=-0,13)$ e pontuação total da escala MIF $(r=-0,20)$ foi negativa em relação à sobrecarga, ou seja, quando a renda mensal, a renda per capita e a pontuação total da escala MIF dos pacientes eram baixas, os valores da pontuação total da escala ZBI de sobrecarga eram altos; indicando que quanto menos independente, quanto menor a renda mensal familiar e a renda per capita forem do paciente, consequentemente mais sobrecarga teria o cuidador informal; entretanto a sobrecarga tinha fraca correlação com essas variáveis analisadas, tendo os valores de $r$ (independente do sinal) próximas de 0 .

\section{Discussão}

Os dados da pesquisa caracterizaram que todos os cuidadores informais eram membros da família e a escolha do cuidador informal estava relacionada com fatores de: grau de parentesco mais próximo (7:16 cônjuges, 4:16 pais, 2:16 filhos), gênero (11:16 feminino), proximidade (14:16 residiam com a pessoa a ser cuidada), tempo de disponibilidade e vontade (11:16 não trabalhavam e 14:16 não estudavam, todos dedicavam os cinco dias da semana e 14:16 dedicavam também os dois dias do final de semana, sendo em média $20 \pm 7 \mathrm{~h} / \mathrm{dia}$ ) e que os cuidadores informais não tinham formação básica orientada (13:16 não tinha curso e nem treinamento para cuidar); sendo condizentes com Azevedo ${ }^{4}$, Gratão ${ }^{6}$ e Moreira ${ }^{3}$, em que a família é quem geralmente assume a responsabilidade pela saúde de seus membros, prestando cuidados e promovendo a saúde, assumindo a responsabilidade de cuidar, sendo a escolha do cuidador informal relacionada com o grau de parentesco (cônjuges, pais e filhos), gênero (maioria feminino), com proximidade de convivência física e afetiva, onde o cuidador principal é referência por maior disponibilidade, instinto, vontade ou capacidade, geralmente sem formação básica orientada.

O grau de sobrecarga dos cuidadores informais demonstrou que a maioria (nove dos 16 cuidadores informais) tinha ausência de sobrecarga, cinco a tinham de leve a moderada e dois de moderado a severa, não havendo nenhum com intenso grau, conforme Tabela 1; indicando que apesar dos cuidadores informais terem que cuidar e lidar com a dependência física e mental dos pacientes neurológicos cadeirantes adultos, eles não estavam sendo intensamente afetados em suas atividades cotidianas, nos relacionamentos sociais e no equilíbrio emocional. Entretanto, apesar de não apresentarem um grau de sobrecarga intensa que necessitaria de alguma intervenção, a maioria (15:16) dos cuidadores informais contava com a ajuda de outras pessoas para

Tabela 5. Resultado da análise entre a sobrecarga dos cuidadores informais e as variáveis quantitativas dos pacientes neurológicos cadeirantes adultos. Itu-SP, Brasil - 2015.

\begin{tabular}{lcc}
\hline & \multicolumn{2}{c}{$\begin{array}{c}\text { Sobrecarga do } \\
\text { Cuidador Informal }\end{array}$} \\
\cline { 2 - 3 } \multicolumn{1}{c}{$\begin{array}{c}\text { Variáveis } \\
\text { Avaliadas }\end{array}$} & $\begin{array}{c}\text { Coeficiente } \\
\text { de Correlação }\end{array}$ & $\begin{array}{c}\text { palor } \\
\text { de Pearson }\end{array}$ \\
\hline Idade (em anos completos) & $\mathrm{r}=0,10$ & 0,72 \\
Tempo como cadeirante (meses) & $\mathrm{r}=0,09$ & 0,75 \\
Renda mensal familiar (R\$) & $\mathrm{r}=0,03$ & 0,92 \\
Renda per capita (R\$) & $\mathrm{r}=0,13$ & 0,64 \\
Escala de Avaliação MIF & $\mathrm{r}=0,20$ & 0,46 \\
\hline
\end{tabular}

* Avaliada pela pontuação total da Escala ZBI ${ }^{9}$; ${ }^{* *}$ Avaliada pela pontuação total da Escala de Avaliação MIF ${ }^{13}$.

Tabela 4. Distribuição da frequência do grau de independência funcional dos pacientes neurológicos cadeirantes adultos, segundo Escala MIF ${ }^{13}$. Itu-SP, Brasil - 2015.

\begin{tabular}{lrr}
\hline \multicolumn{1}{c}{ Variável Avaliada } & fi & \% \\
\hline Grau de Independência Funcional $^{*}$ & & \\
Dependência Total (18 pontos) & 0 & 0,00 \\
Dependência Máxima a Moderada (19 a 60 pontos) & 12 & 75,00 \\
Independência Mínima a Supervisão, Estímulo ou Preparo (61 a 103 pontos) $^{\text {Independência Modificada a Independência Completa (104 a 126 pontos) }}$ & 4 & 0 \\
Total & 16 & 0,00 \\
\hline
\end{tabular}

*Avaliada pela pontuação total da Escala de Avaliação MIF ${ }^{13}$. 
cuidar, sendo esta solicitada apenas a parentes próximos. Outro ponto a ser observado é que as pessoas a serem cuidadas, ou seja, esses dependentes de cuidados, passavam também pelo atendimento, tratamento e tinham orientações na Clinica/Escola do CEUNSP; e que de acordo com Camargos $^{10}$ o suporte terapêutico e social dado por instituições de apoio, neste caso a Clínica/Escola de Fisioterapia da CEUNSP, podem ter auxiliado a família em estratégias de enfrentamento de determinadas situações, transpondo de forma mais tranquila e influenciando positivamente na percepção da sobrecarga destes cuidadores.

Ainda, o estudo apresentou associação da sobrecarga do cuidador informal em relação ao seu nível de conhecimento sobre a patologia acometida ( $\mathrm{p}$-valor $=0,01)$ e o seu grau de escolaridade ( $p$-valor $=0,01)$, havendo maior sobrecarga entre os cuidadores com menor nível de conhecimento (pouco e insuficiente informação/pouco e suficiente informação) em comparação com maiores níveis de conhecimento (bem informados), e maior sobrecarga nos cuidadores com baixo grau de escolaridade ( 1 a 4 anos de estudo) em comparação com médio e alto grau de escolaridade (5 a 8 anos e 13 ou mais anos de estudo), sendo os dados condizentes com Azevedo ${ }^{4}$, que indicou estudos de revisão da literatura nos quais os resultados apontavam sobrecarga do cuidador informal agravada pela falta de informação a respeito da doença do paciente, do tratamento e das estratégias mais adequadas para lidar com os problemas dos sujeitos cuidados.

Apesar da maioria, 12 dos 16 pacientes neurológicos cadeirantes adultos, ter grau de independência funcional baixa, segundo pontuação da Escala de Avaliação MIF, com dependência máxima a moderada, conforme Tabela 4, e que provavelmente por conta disto os cuidadores informais tinham que se dispor em média $20 \pm 7$ horas por dia, com tempo mínimo de 6 horas/dia e máximo de 24 horas/dia para cuidar na semana e praticamente nos finais de semana, verificouse que isso não apresentou uma associação com a sobrecarga, indicando uma correlação fraca. É importante neste sentido destacar que o cuidador e a pessoa a ser cuidada, aparentavam ter consciência da função do primeiro, que era acompanhar e auxiliar a pessoa cuidada (indivíduo dependente) fazendo somente as atividades que ela não conseguia realizar sozinha. E que de certa forma, demonstrou que ambas buscavam formas para planejar e adequar suas rotinas, compreen- dendo um ao outro, sem tirar suas autonomias, focando numa relação boa de convivência; talvez isso se deva em parte pelo grau de escolaridade dos cuidadores ser elevado em comparação aos dependentes de cuidados e da idade média elevada de ambos os grupos, mas não podemos afirmar ao certo, pois não foi este o intuito da análise do estudo.

A pesquisa não apresentou associação da sobrecarga em relação às outras variáveis analisadas e indicou fraca correlação também da sobrecarga do cuidador informal com: a idade (do cuidador informal e do paciente neurológico cadeirante adulto), a questão econômica (renda mensal familiar e per capita do paciente neurológico cadeirante adulto) e tempo (como cuidador e cadeirante).

\section{Considerações finais}

Os resultados obtidos neste estudo não podem ser generalizados uma vez que a amostra estudada foi pequena e restrita a cuidadores informais de pacientes neurológicos cadeirantes adultos que frequentavam programas de reabilitação. Ao analisarmos estes dados, não podemos descartar a possibilidade dos entrevistados terem ficado com algum receio, ao responder a Escala ZBI, devido a fatores como: grau de religiosidade, grau de instrução do cuidador informal, por serem membros próximos e da família dos sujeitos cuidados. Entretanto, o estudo apontou uma relação de compreensão e entendimento da situação entre ambas as partes, tanto do cuidador informal como do sujeito cuidado, talvez pela familiaridade ou intimidade entre elas, até por muitas vezes a função de cuidador informal não ser considerada um encargo para muitos parentes, sendo uma tarefa normal para os pais; mas que não podemos afirmar ao certo, pois este não foi o objetivo e o intuito de investigação do presente estudo, sendo necessários maiores pesquisas nesta área.

Por fim, o suporte terapêutico e social dado pelas instituições de apoio, como a Clínica/Escola de Fisioterapia da CEUNSP, demonstra ser importante para auxiliar a família e os cuidadores informais a enfrentar situações de forma mais tranquila, influenciando na melhor compreensão da patologia acometida, no conhecimento de como cuidar, tratar e lidar com essas questões no dia a dia, até pelo convívio com outras pessoas que enfrentam o mesmo problema. 


\section{Colaboradores}

LC Boaventura, HC Borges e AH Ozaki participaram igualmente na elaboração do artigo: na pesquisa; objetivos e metodologia; concepção, análise e interpretação dos dados; redação e revisão crítica; e na aprovação final do manuscrito.

\section{Agradecimentos}

Agradecemos a coordenação e o corpo docente da Clínica/Escola de Fisioterapia do CEUNSP pela estrutura e o apoio para a realização dessa pesquisa.

\section{Referências}

1. Brasil. Ministério da Saúde (MS). Secretaria de Atenção à Saúde, Secretaria de Gestão do Trabalho e da Educação na Saúde. Guia prático do cuidador Série A Normas e Manuais Técnicos. Brasília: MS; 2008.

2. Brasil. Ministério da Previdência e Assistência Social. Secretaria de Assistência Social. Publicação do papel do cuidador domiciliar. São Paulo: Instituto de Estudos Especiais PUC-SP; 1998.

3. Moreira IMPB. O Doente Terminal em Contexto Familiar: uma análise da experiência de cuidar vivenciada pela família. 2a ed. Coimbra: Formasau; 2006.

4. Azevedo RS. Sobrecarga do cuidador informal da pessoa idosa frágil: uma revisão sistemática [dissertação]. Belo Horizonte: UFMG; 2010.

5. Brasil. Ministério da Saúde (MS). Secretaria de Atenção à Saúde. Caderno de Atenção Básica no 19 Série A Normas e Manuais: Técnicos de Envelhecimento e saúde da pessoa idosa. Brasília: MS; 2006.

6. Gratão ACM. Sobrecarga vivenciada por cuidadores idosos na comunidade [tese]. Ribeirão Preto: USP; 2010.

7. Santos AAS, Vargas MM, Oliveira CCC, Macedo IAB. Avaliação da sobrecarga dos cuidadores de crianças com paralisia cerebral. Ciênc. Cuid. Saúde 2010; 9(3):503-509.

8. Sebastiao SG, Gilberto BC, Antonio LT. O exame neurológico - bases anatomofuncionais. 2a ed. São Paulo: Revinter; 2007.

9. Scazufca M. Brazilian version of the Burden Interview scale for the assessment of burden of care in carers of people with mental illnesses. Res. Bras. Psiquiatr. 2002; 24(1):12-17.

10. Camargos ACR, Lacerda TTB, Viana SO, Pinto LRA, Fonseca MLS. Avaliação da sobrecarga do cuidador de crianças com paralisia cerebral através da escala Burden Interview. Res. Bras. Mater. Saúde. Infant. 2009; 9(1):31-37.
11. Hébert R, Bravo G, Préville M. Reliability, validity and reference values of the Zarit Burden Interview for assessing informal caregivers of community-dwelling older persons with dementia. Canadian Journal of Aging 2000; 19(4):494-507.

12. Luzardo AR, Gorini MIPC, Silva APSS. Características de idosos com doença de Alzheimer e seus cuidadores: uma série de casos em um serviço de neurogeriatria. Texto e Contexto de Enfermagem 2006; 15(4):587-594.

13. Riberto M, Miyazaki MH, Jucá SSH, Sakamoto H, Pinto PPN, Battistella LR. Validação da versão brasileira da Medida de Independência Funcional. Acta Fisiátrica 2004; 11(2):72-76.

14. Levin J, Fox JA. Estatística para Ciências Humanas. 9a ed. São Paulo: Prentice Hall; 2004.

15. Dancey C, Reidy J. Estatística Sem Matemática para Psicologia: Usando SPSS para Windows. $3^{\text {a }}$ ed. Porto Alegre: Artmed; 2006.

16. Figueiredo DBF, Silva JAJ. Desvendando os mistérios do coeficiente de correlação de Pearson(r). Rev Política Hoje 2009; 18(1):115-146.

17. Portal Action [homepage]. Portal Action: Manual Action. [acessado 2 dez 2014]. Disponível em: http:// www.portalaction.com.br/manual-action

Artigo apresentado em 10/03/2016

Aprovado em 28/06/2016

Versão final apresentada em 30/06/2016 